\title{
Synthesis of imides based on phenylcycloalkanedicarboxylic acids and derivatives trifluoromethylaniline
}

\author{
(C) Anna A. Koverda, ${ }^{1}{ }^{+}$Mikhail N. Koverda, ${ }^{2}$ Valeria D. Bulanina, ${ }^{1}$ \\ Alexandra I. Korshunova, ${ }^{1}$ and Angelina S. Danilova ${ }^{1}$ \\ ${ }^{1}$ Organic and Analitical Chemistry Division. Yaroslavl State Technical University. Moscovsky Ave., 88. \\ Yaroslavl, 150023. Russia. Phone: +7 (4852) 44-05-29.E-mail: a.koverda@pm.me \\ ${ }^{2}$ Yaroslavl State Institute of Raw Materials and Food Quality. Moscovsky Ave., 76 . \\ Yaroslavl, 150030.Russia.Phone: +7 (4852) 47-86-86.E-mail: m.kov@pm.me
}

*Supervising author; ${ }^{+}$Corresponding author Keywords: phenylcycloalkanedicarboxylic acids, trifluoromethyl group, aromatic amines, imidization.

\begin{abstract}
Previously it was shown that the stereochemical result of the alkylation reaction of benzene with cycloalkanedicarboxylic acids depends on the order of mixing the reagents. Based on this, we have developed methods for the synthesis of phenylcycloalkanedicarboxylic acids and methods for the separation of their isomers. The resulting diastereomerically pure compounds were used to synthesize new imides containing the trifluoromethyl group, which are potentially biologically active substances with antitumor properties.

Since aromatic amines containing the $\mathrm{CF}_{3}$ group are weak nucleophiles, during the reaction both complete and incomplete closure of the imide cycle can be observed. The research problem was to study the dependence of the character of the reaction products and their ratio (imide/amides) on the flow conditions: the solvent used and the use of auxiliary reagents. When carrying out the imidization reaction in acetic acid, the formation of a mixture of imide and isomeric amides is observed, and the use of tea in toluene or DMF and in the system of acetic acid - pyridine, taken in a $2: 3$ ratio, leads to the formation of an imide only, but with different yields. Were tested methods for obtaining imides in pyridine through the stage of obtaining dichlorohydrin, which were obtained using thionyl chloride and using triphenylphosphine - carbon tetrachloride. Imides were also obtained in good yields in one-pot reactions by heating a mixture of pyridine, acid, amine, and thionyl chloride.

The structure of the new compounds was confirmed by IR spectroscopy, ${ }^{1} \mathrm{H},{ }^{13} \mathrm{C}$ NMR spectra, capillary electrophoresis, elemental analysis and HPLC.

\section{References}

[1] C.Et. Ferroni, all. 1,4-Substituted Triazoles as Nonsteroidal Anti-Androgens for Prostate Cancer Treatment. Journal of Medicinal Chemistry. 2017. Vol.60. P.3082-3093.

[2] M.S alvati, Et. all. Structure based approach to the design of bicyclic-1H-isoindole-1,3(2H)-dione based androgen receptor antagonists. Bioorganic \& Medicinal Chemistry Letters. 2005. Vol.15. P.271-276.

[3] M.E. Salvati, Et. all. Fused heterocyclic succinimidecompounds and analogs thereof, modulators of nuclear hormone receptor function, Patent WO, no. 03/062241 (A1), 2003.

[4] I.N. Tarabara, Et all. Synthesis and neurotropic activity of endic anhydride derivatives. Journal of Organic and Pharmaceutical Chemistry [in Ukraine]. 2006. Vol.4. No.2. P.54-59.

[5] A.A. Koverda, M.N. Koverda, E.R. Kofanov, and G.G. Krasovskaya. Stereoselectivity of alkylation benzene by $(1 R, 2 S) /(1 S, 2 R)$-cyclohex-4-ene-1,2-dicarboxylic acid and $(1 R, 2 S) /(1 S, 2 R)-4$ methylcyclohex-4-ene-1,2-dicarboxylic acid. Butlerov Communications. 2018. Vol.55. No.9. P.106-114. DOI: $10.37952 /$ ROI-jbc-01/18-55-9-106

[6] Y.V. Karjakin. Pure chemical substances. Moscow: Chemistry. 1974. (russian)
\end{abstract}

